\title{
A NEW LARGE DEVIATION INEQUALITY FOR U-STATISTICS OF ORDER 2
}

\author{
JEAN BREtaGnOLle ${ }^{1}$
}

\begin{abstract}
We prove a new large deviation inequality with applications when projecting a density on a wavelet basis.

Résumé. Nous prouvons une inégalité de grandes déviations applicable à la projection d'une densité sur une base d'ondelettes.
\end{abstract}

AMS Subject Classification. 60F10, 62-xx.

Received July 2, 1998. Revised September 14, 1999.

\section{INTRODUCTION AND MAIN RESULT}

Let $F$ be some law on $\mathbb{R}$. When $g$ is a measurable function from $\mathbb{R}^{d}$ to $\mathbb{R}, E(g)$ and $\operatorname{Var}(g)$ denote expectation and variance with respect to the Probability $F^{\otimes d}$. Let $f$ be a bounded and symmetric function from $\mathbb{R}^{2}$ to $\mathbb{R}$. Following Arcones and Giné [1], we construct its canonical projections: $\xi$ and $\eta$ being independent with law $F$

$$
\begin{aligned}
\pi_{1} f(x) & =E f(x, \eta)-E f(\xi, \eta) . \\
\pi_{2} f(x, y) & =f(x, y)-E f(x, \eta)-E f(\xi, y)+E f(\xi, \eta) .
\end{aligned}
$$

Let $\xi_{i}, i=1,2, \cdots, n$ be a $n$-sample of $F(n \geq 2)$. We consider the $U$-statistics (without any normalisation)

$$
\begin{aligned}
U_{n}^{(2)}(f) & =\Sigma_{1 \leq i \neq j \leq n} f\left(\xi_{i}, \xi_{j}\right) . \\
U_{n}^{(1)}\left(\pi_{1} f\right) & =\Sigma_{1 \leq i \leq n} \pi_{1} f\left(\xi_{i}\right) . \\
U_{n}^{(2)}\left(\pi_{2} f\right) & =\Sigma_{1 \leq i \neq j \leq n} \pi_{2} f\left(\xi_{i}, \xi_{j}\right), \text { thus } \\
\left.U_{n}^{(2)}(f-E f)\right) & =2(n-1) U_{n}^{(1)}\left(\pi_{1} f\right)+U_{n}^{(2)}\left(\pi_{2} f\right) .
\end{aligned}
$$

We are interested in a large deviation inequality for the latter $U$-statistic when $f$ is centered and bounded.

First, if $|f| \leq c$ and $E f^{2}=\sigma^{2}$, the usual Bernstein type inequality is

$$
P\left(U_{n}^{(2)}(f-E f) \geq n(n-1) t\right) \leq \exp \left(-[n / 2] t^{2} /\left\{2 \sigma^{2}+2 c t / 3\right\}\right) .
$$

But we can consider $U_{n}^{(1)}\left(\pi_{1} f\right)$ (which is a sum of i.i.d. $\mathbb{R}$-valued random variables) as the main part and it can be interesting to bound separately the second part $U_{n}^{(2)}\left(\pi_{2} f\right)$. Now, as $\pi_{2} f$ is canonical of order 2 , if $\left|\pi_{2} f\right| \leq c$

Keywords and phrases: Large deviations, U-statistics.

1 URAD 0743, Université d'Orsay, bâtiment 425, 91405 Orsay Cedex, France; e-mail: Jean.Bretagnolle@math.u-psud.fr 
and $\sigma^{2}=E\left(\pi_{2} f\right)^{2}$, there exist two constants $c_{1}, c_{2}$ such that

$$
P\left(\left|U_{n}^{(2)}\left(\pi_{2} f\right)\right| \geq(n-1) t\right) \leq c_{1} \exp \left(-c_{2} t /\left\{\sigma+c^{2 / 3} t^{1 / 3} n^{-1 / 3}\right\}\right)
$$

((AG1) and (AG2) can be found in Arcones and Giné [1]).

The normalized version of inequality (AG2) can be restated

$$
\left.P\left(\left|U_{n}^{(2)}\left(\pi_{2} f\right)\right|\right) \geq a_{3} \operatorname{Max}\left(n \sigma x, c \sqrt{n} x^{3 / 2}\right)\right) \leq a_{4} \exp (-x) .
$$

The aim of the paper is to give a new large deviation inequality (Th. 1 and Cor. 1 later). To every partition $D$ we associate two functionnals $\|f\|_{D}$ and $v_{D}(F)$ (see Def. 4 later) such that

$$
P\left(\left|U_{n}^{(2)}\left(\pi_{2} f\right)\right| \geq a_{3}^{\prime} x\|f\|_{D} \operatorname{Max}\left(n \sqrt{v_{D}(F)}, 1\right)\right) \leq a_{4}^{\prime} \exp (-2 \sqrt{x})
$$

where all $a_{3}, a_{3}^{\prime}$ are universal constants. For a comparison between the two inequalities, see the discussion after Theorem 2 infra.

We need now some definitions: let $\left(I_{\lambda} \mid \lambda \in D\right)$ be a Borelian partition of $\mathbb{R}$ finite or enumerable, where $I_{\lambda}$ denotes the subset and its indicator. Let $\tau$ be a permutation of $D$. Its graph $g_{\tau}$ is $\{(\lambda, \tau(\lambda)) \mid \lambda \in D\}$, a subset of $D \times D$. A collection $G$ is an enumerable set $\left(\tau_{s} \mid s \in G\right)$ of permutations such that $D \times D \subset \cup_{s \in G} g_{\tau_{s}}$. $\mathbb{H}$ is the family of collections. For any matrix $M=\left(a_{\lambda, \mu} \mid \lambda \in D ; \mu \in D\right)$ we set

$$
\|M\|_{G}=\Sigma_{i \in G} \operatorname{Sup}_{\lambda \in D}\left|a_{\lambda, \tau_{i}(\lambda)}\right| \text { and }\|M\|_{D}=\operatorname{Inf}_{G \in \mathbb{H}}\|M\|_{G} .
$$

Let $f$ be a bounded real valued function defined on $\mathbb{R} \times \mathbb{R}$. We set

$$
\begin{aligned}
M(D, f) & =\left(a_{\lambda, \mu}\right) \text { where } a_{\lambda, \mu}=\operatorname{Sup}\left(f^{2}(x, y) I_{\lambda}(x) I_{\mu}(y)\right) \\
\|f\|_{D}^{2} & =\|M(D, f)\|_{D} \\
v_{D}(F) & =\Sigma_{\lambda} P\left(\xi \in I_{\lambda}\right)^{2} .
\end{aligned}
$$

Our main result is

Theorem 1. There exists some constant $C(C=80 \pi$ holds) such that for every integer $k>0$, for every partition $D$, for every symmetric $f$

$$
E\left(U_{n}^{(2)}\left(\pi_{2} f\right)^{2 k}\right) \leq C^{2 k} \times\left(E \mathcal{N}^{2 k}\right)^{4} \times\|f\|_{D}^{2 k} \operatorname{Max}\left(n^{2 k}\left(v_{D}(F)\right)^{k}, n^{2} v_{D}(F)\right) .
$$

where $\mathcal{N}$ denotes the standard normal distribution.

Corollary 1. There exists some constant $C$ such that for every partition $D$, for every symmetric $f$, for every $x>0$

$$
P\left(\left|U_{n}^{(2)}\left(\pi_{2} f\right)\right| \geq C x\|f\|_{D} \operatorname{Max}\left(n \sqrt{v_{D}(F)}, 1\right)\right) \leq \exp (6-2 \sqrt{x}) .
$$

As we will see in the following Discussion,

Remark 1: The classical inequality (AG) is strictly better whenever $x \leq n \sigma^{2} / c^{2}$.

Remark 2: Nevertheless, our inequality can work when the classical one does not.

Remark 3: Finally, up to some logarithm, $\sqrt{x}$ is the best possible rate.

Main application: Let $\left(\Psi_{1}, \Psi\right)$ be a wavelet and $\varepsilon_{\lambda}, \varepsilon_{\ell, \lambda}$ be the associated basis:

$$
\varepsilon_{\lambda}(x)=\Psi_{1}(x+\lambda) /\left\|\Psi_{1}\right\|_{2}, \varepsilon_{\ell, \lambda}(x)=2^{\ell / 2} \Psi\left(2^{\ell} x+\lambda\right) /\|\Psi\|_{2}(\lambda \in \mathbb{Z}, \ell \in \mathbb{N}) .
$$


Let $p$ be some density of Probability on $\mathbb{R}$ equipped with Lebesgue measure $d x$, assumed to be square integrable in applications.

Let $\beta_{\lambda}$ be $\int p(x) \varepsilon_{\lambda}(x) d x$ and $\gamma_{\ell, \lambda}$ be $\int \varepsilon_{\ell, \lambda}(x) p(x) d x$.

We set $p_{L}=\Sigma_{\lambda} \beta_{\lambda} \varepsilon_{\lambda}+\Sigma_{\ell \leq L} \Sigma_{\lambda} \gamma_{\ell, \lambda} \varepsilon_{\ell, \lambda}$ (the projection of $p$ up to level of resolution $L$ ) and want to estimate its square norm

$$
\left.\theta_{L} \doteq\left\|p_{L}\right\|_{2}^{2}=\Sigma_{\lambda} \beta_{\lambda}^{2}+\Sigma_{\ell \leq L}\left(\Sigma_{\lambda} \gamma_{\ell, \lambda}^{2}\right)\right)
$$

When $\left(\xi_{i} \mid 1 \leq i \leq n\right)$ is a $n$-sample with density $p(n \geq 2)$, the empirical estimators of coefficients are $\beta_{\lambda, n}=\Sigma_{i} \varepsilon_{\lambda}\left(\xi_{i}\right) / n, \gamma_{\ell, \lambda, n}=\Sigma_{i} \varepsilon_{\ell, \lambda}\left(\xi_{i}\right) / n$. Let $p_{L, n}$ be

$$
p_{L, n}=\Sigma_{\lambda} \beta_{\lambda, n} \varepsilon_{\lambda}+\Sigma_{\ell \leq L} \Sigma_{\lambda} \gamma_{\ell, \lambda, n} \varepsilon_{\ell, \lambda} .
$$

The "natural estimator" for $\theta_{L}$ is

$$
\Sigma_{\lambda} \beta_{\lambda, n}^{2}+\Sigma_{\ell \leq L}\left(\Sigma_{\lambda} \gamma_{\ell, \lambda, n}^{2}\right)
$$

But the latter has positive bias, and the unbiased estimator is

$$
\widehat{\theta}_{L, n}=(n(n-1))^{-1} \Sigma_{1 \leq i \neq j \leq n}\left\{\Sigma_{\lambda} \varepsilon_{\lambda}\left(\xi_{i}\right) \varepsilon_{\lambda}\left(\xi_{j}\right)+\Sigma_{\ell \leq L}\left(\Sigma_{\lambda} \varepsilon_{\ell, \lambda}\left(\xi_{i}\right) \varepsilon_{\ell, \lambda}\left(\xi_{j}\right)\right)\right\} .
$$

Let $\Delta_{n, L}=\widehat{\theta}_{L, n}-\theta_{L}$. According to (5), $\Delta_{n, L}$ can be decomposed into canonical $U$-statistics in the following way: let $\Phi_{1}$ be $\Psi_{1} /\left\|\Psi_{1}\right\|_{2}$ and let $\Phi$ be $\Psi /\|\Psi\|_{2}$,

$$
\begin{aligned}
f(x, y) & =\Sigma_{\lambda} \Phi_{1}(x+\lambda) \Phi_{1}(y+\lambda), f_{\ell}(x, y)=\Sigma_{\lambda} \Phi\left(2^{\ell} x+\lambda\right) \Phi\left(2^{\ell} y+\lambda\right) \\
\delta_{n} & =(2 / n) U_{n}^{(1)}\left(\pi_{1} f\right)+(1 / n(n-1)) U_{n}^{(2)}\left(\pi_{2} f\right) \\
\delta_{\ell, n} & =\left(2 \cdot 2^{\ell} / n\right) U_{n}^{(1)}\left(\pi_{1} f_{\ell}\right)+\left(2^{\ell} / n(n-1)\right) U_{n}^{(2)}\left(\pi_{2} f_{\ell}\right) .
\end{aligned}
$$

Then $\Delta_{n, L}=\delta_{n}+\Sigma_{\ell \leq L} \delta_{\ell, n}$. In the decomposition above, the sum of $U$-statistics of order 1 is equal to the part up to level of resolution $L$ of $\int p\left(p_{n}-p\right)$. It can be bounded in Probability by classical Bernstein's inequality. The control of each $U$-statistic of order 2 will be performed by our inequality. This is very useful, either to estimate $\theta_{L}$ [3], or in model selection: in this problem, the authors consider a wide family of finite dimensional projections. Our study is quite general, but we observe that if $\Psi_{1}$ and $\Psi$ are with compact support, for every $L$ the family $\left(\beta_{\lambda}, \alpha_{\ell, \lambda} \mid \ell \leq L\right)$ is in fact with finite dimension, and hypothesis $(\mathrm{H})$ infra holds. Thus our result can be used in adaptive estimation of quadratic functionals in a density model (see for example [4] where white noise is treated), where the theory needs good bounds up to $2^{\ell}=O\left(n^{2}\right)$. These bounds cannot be obtained by classical Hoeffding's bounds (see the chapter "Discussion").

We assume in the whole paper

\section{Hypothesis (H).}

$$
\begin{aligned}
& \qquad|\Phi(x)| \leq \Sigma_{u \in \mathbb{Z}} \omega_{u} 1_{u \leq x<u+1} \text { with } \Sigma_{u \in \mathbb{Z}} \omega_{u}<\infty \\
& \text { and we set } M^{2}(\Phi)=\Sigma_{(u, v, w) \in \mathbb{Z}^{3}} \omega_{u} \omega_{v} \omega_{u+w} \omega_{v+w} .
\end{aligned}
$$

In the particular case when the law has density $p$, for the normalised $U$-statistic, we have

Theorem 2. We assume that the function $\Phi$ with $\|\Phi\|_{2}=1$ satisfies hypothesis $(H)$, and that the law $F$ has a density $p$ with $\|p\|_{2}=\left(\int p^{2}(x) d x\right)^{1 / 2}<\infty$. Set

$$
\|p\|_{2, \ell}=2^{\ell / 2}\left[\Sigma_{k \in \mathbb{Z}}\left(\int_{k 2^{-\ell}}^{(k+1) 2^{-\ell}} p(x) d x\right)^{2}\right]^{1 / 2}
$$




$$
\begin{gathered}
\delta_{\ell, n}^{(2)}=\left(2^{\ell} / n(n-1)\right) U_{n}^{(2)}\left(\pi_{2} f_{\ell}\right) \text { where } f_{\ell}(x, y)=\Sigma_{\lambda} \Phi\left(2^{\ell} x+\lambda\right) \Phi\left(2^{\ell} y+\lambda\right) \\
Z_{\ell, n}=\sqrt{n(n-1)} 2^{-\ell / 2} \delta_{\ell, n}^{(2)} .
\end{gathered}
$$

Then, if $n\|p\|_{2, \ell} \geq 2^{\ell / 2}$, we have, for $C$ as in Theorem 1

$$
P\left(\left|Z_{\ell, n}\right| \geq 2 C \times M(\Phi) \times x\right) \leq \exp (6-2 \sqrt{x}) .
$$

In this formula, $\operatorname{Var}\left(Z_{\ell, n}\right)$ does not depend on $n$. Moreover, there exists positive and finite constants a $(\Phi), b(\Phi)$ depending only on $\Phi$ such that

$$
\begin{gathered}
\lim _{\ell \rightarrow \infty}\|p\|_{2, \ell}=\|p\|_{2} . \\
\lim _{\ell \rightarrow \infty}\left\|\pi_{2} f_{\ell}\right\|_{\infty}=b(\Phi) \text { and } a(\Phi)\|p\|_{2}^{2} \leq \liminf _{\ell \rightarrow \infty} \operatorname{Var}\left(Z_{\ell, n}\right) . \\
\left\|\pi_{2} f_{\ell}\right\|_{\infty} \leq 4 M(\Phi) \text { and } \operatorname{Var}\left(Z_{\ell, n}\right) \leq\|p\|_{2}^{2} M^{2}(\Phi) .
\end{gathered}
$$

Remark 4: Such a result is interesting only if it works, for given $n, \ell$, uniformly for large classes of densities. Obviously we need some uniform control of $\|p\|_{2}$, but this is not sufficient in view of condition $n\|p\|_{2, \ell} \geq 2^{\ell / 2}$.

Thus we need some extra condition. If for example we assumme that the support of $p$ is contained in some interval $[x, x+M]$ we get $\|p\|_{2, \ell} \geq 1 /(M+1)$ and the bound works if $n^{2} \geq(M+1) 2^{\ell}$.

I would like to thanks the two anonymous referees whose remarks and suggestions have much improved the presentation.

\section{Discussion}

a) About Corollary 1 .

We consider inequality stated in Corollary 1 and assertion (AG2"). We assumme $\|f\|_{\infty}=1$. Without further knowledge about the law $F$, we can only bound $\sigma^{2}=\operatorname{Var}\left(\pi_{2}(f)\right)$ by $E f^{2}$ and $c=\left\|\pi_{2} f\right\|_{\infty}$ by 4 . Up to some change of $a_{3},(\mathrm{AG} 2$ ') is restated

$$
P\left(\left|U_{n}^{(2)}\left(\pi_{2} f\right)\right| \geq a_{3} \operatorname{Max}\left(n x \sqrt{E f^{2}}, \sqrt{n} x^{3 / 2}\right)\right) \leq a_{4} \exp (-x)
$$

On the other hand, we have the obvious inequality

$$
E f^{2} \leq\|f\|_{D}^{2} v_{D}(F) .
$$

Proof of Remark 1: Let us assumme $x \leq n \sigma^{2}$. Thus $\operatorname{Max}\left(n \sigma x, c \sqrt{n} x^{3 / 2}\right)=n \sigma x \leq n\|f\|_{2} x \leq x\|f\|_{D} \operatorname{Max}$ $\left(n \sqrt{v_{D}(F)}, 1\right)$ and, up to constants (AG2') is always better than the bound of Corollary 1 whenever $x \leq n \sigma^{2}$.

Nevertheless, our inequality provides a possible alternative if there exists some partition $D$ such that $n^{2} v_{D} \geq 1$ and $\|f\|_{D}^{2} v_{D}(F) \approx E f^{2}$ (up to constant). We exhibit two extremal cases in the case when the law is the uniform one:

1) $f(x, y)=\mathbb{1}_{0 \leq x<p ; 0 \leq y<p}$, where $0<p<1$. We set $q=1-p$. We have $\|f\|_{\infty}=1, E f^{2}=p^{2}$. Choosing $\left.\left.I_{1}=(0, p) I_{\lambda}=\right) p+(\lambda-2) q / K, p+(\lambda-1) q / K\right)$ for $1<\lambda \leq K+1$, we get $v_{D}=p^{2}+q^{2} / K$ and, for a convenient choice of $G,\|f\|_{D}^{2}=1$. Moreover $\operatorname{Var}\left(\pi_{2} f\right)=p^{2} q^{2}$ and $\left\|\pi_{2} f\right\|_{\infty}=\max \left(p^{2}, q^{2}\right)$.

When $p$ is small, up to constants, in the classical inequality we can use either precise true parameters or rough estimates $\left(\|f\|_{\infty}\right.$ and $E f^{2}$ for $\left\|\pi_{2} f\right\|_{\infty}$ and $\left.\operatorname{Var}\left(\pi_{2} f\right)\right)$ and we get

$$
P\left(\left|U_{n}^{(2)}\left(\pi_{2} f\right)\right| \geq a \operatorname{Max}\left(n x p, \sqrt{n} x^{3 / 2}\right)\right) \leq a_{4} \exp (-x)
$$

Our result is

$$
P\left(\left|U_{n}^{(2)}\left(\pi_{2} f\right)\right| \geq C x \operatorname{Max}(n p, 1)\right) \leq \exp (6-2 \sqrt{x})
$$


In this setting, we can assume $x>1, n$ large.

If $1<x<n p^{2}$, the classical inequality is better, but (up to constants) only with respect to the exponent of $x$.

A contrario the classical result does not work in the case when $n p^{2}=o(1)$ but not our one provided that $n p$ is large, and this justify the Remark 2 .

We will see that it is a quite general result in the main application.

Remark 3: Assuming $n p=1$ and denoting $f_{n}$ the corresponding function, when $n \rightarrow \infty$, it is easy to prove that $U_{n}^{(2)}\left(\pi_{2} f_{n}\right)$ converges in law to $Y^{2}-3 Y+1$ where the law of $Y$ is the Poisson law with parameter 1 . Thus

$$
\liminf { }_{x \rightarrow \infty} \lim _{n}\left\{-\log P\left(U_{n}^{(2)}\left(\pi_{2} f_{n}\right) \geq x\right) / \sqrt{x} \log (\sqrt{x})\right\} \geq 1
$$

proving that the power $1 / 2$ is the best possible.

2) Let be $g(x, y)=\mathbb{1}_{0 \leq x \leq 1 ; 0 \leq y \leq 1}-\mathbb{1}_{p \leq x \leq 1-p ; p \leq y \leq 1-p}(p$ small $)$ and $\varepsilon(x)=\mathbb{1}_{x \leq 1 / 2}-\mathbb{1}_{1 / 2<x}$ and finally $f(x, y)=\varepsilon(x) \varepsilon(y) g(x, y)$. Then, for the uniform law, $f=\pi_{2} f$ and $\operatorname{Var}\left(\pi_{2} f\right)=\operatorname{Var}(f)=4 p(1-p)$. Obviously, for every $D,\|f\|_{D}^{2}=|D|$ and $v_{D}(F) \geq 1 / D$, thus $\|f\|_{D} \max \left(n \sqrt{v_{D}(F)}, 1\right) \geq n$ (and $n$ is obtained by the partition with one element).

The classical inequality gives for some universal $a$

$$
P\left(\left|U_{n}^{(2)}\left(\pi_{2} f\right)\right| \geq a \operatorname{Max}\left(n \sqrt{p} x, \sqrt{n} x^{3 / 2}\right)\right) \leq a_{4} \exp (-x)
$$

and, whatever be $p$, our inequality provides only

$$
P\left(\left|U_{n}^{(2)}\left(\pi_{2} f\right)\right| \geq C x n\right) \leq \exp (6-2 \sqrt{x})
$$

a very poor result!

b) About the main application:

b1) We consider firstly the case of the Haar basis $\left(\Phi(x)=\mathbb{1}_{0 \leq x<1}\right.$ or $\left.\Phi(x)=\mathbb{1}_{0 \leq x<1 / 2}-\mathbb{1}_{1 / 2 \leq x<1}\right)$, with uniform law on the interval ]0,1]. In the first case, at the level $\ell$, setting $D=\left(\left[\lambda 2^{-\ell},(\lambda+1) 2^{-\ell}[\mid \lambda \in \mathbb{Z})\right.\right.$, $f_{\ell}=\Sigma_{\lambda \in \mathbb{Z}} \Phi\left(2^{\ell} x+\lambda\right) \Phi\left(2^{\ell} y+\lambda\right)$, we have $M(\Phi)=\left\|f_{\ell}\right\|_{D}=1,\left\|\pi_{2}\left(f_{\ell}\right)\right\|_{\infty}=\left(1-2^{-\ell}\right), v_{D}(F)=2^{-\ell}$ and $\operatorname{Var}\left(\pi_{2}\left(f_{\ell}\right)\right)=\left(1-2^{-\ell}\right)^{2} 2^{-\ell}$.

Thus for every $\ell \geq 1$ we have

$$
\begin{gathered}
2^{\ell} v_{D}(F)=\|f\|_{D}=1 . \\
1 / 2 \leq \operatorname{Var}\left(Z_{\ell, n}\right)=2^{\ell} \operatorname{Var}\left(\pi_{2} f_{\ell}\right) \leq 1 . \\
1 / 2 \leq\left\|\pi_{2} f_{\ell}\right\|_{\infty} \leq 1
\end{gathered}
$$

and, whenever $2^{\ell} \leq n^{2}$, Theorem 2 provides

$$
P\left(\left|Z_{\ell}\right| / \sqrt{\operatorname{Var}\left(Z_{\ell, n}\right)} \geq 2 C x\right) \leq \exp (6-2 \sqrt{x})
$$

The classical one provides

$$
P\left(\left|Z_{\ell}\right| / \sqrt{\operatorname{Var}\left(Z_{\ell, n}\right)} \geq a_{3}\left(x+\left(2^{\ell} / n\right)^{1 / 2} x^{3 / 2}\right)\right) \leq a_{4} \exp (-x)
$$

and does not work if $n=o\left(2^{\ell}\right)$.

Remark 5: Massart (private communication) thinks that using Talagrand's inequality the best possible bandwith is $2^{\ell}=O\left(n^{3 / 2}\right)$ and this is the principal motivation of this work. 
b2) Finally, let $\kappa_{n, \ell}^{2}$ the chi-square (with $2^{\ell}-1$ degrees of freedom) associated to the partition: with $N_{\lambda}=$ $\Sigma_{1 \leq i \leq n} \mathbb{1}_{\lambda 2^{-\ell}<\xi_{i} \leq(\lambda+1) 2^{-\ell}}$

$$
\kappa_{n, \ell}^{2}=\Sigma_{\lambda}\left(N_{\lambda}-E N_{\lambda}\right)^{2} / E N_{\lambda} .
$$

The centered and normalised $\kappa_{n, \ell}^{2}$ is equal to $Z_{\ell} / \sqrt{\operatorname{Var}\left(Z_{\ell, n}\right)}$. Thus our result provides a large deviation inequality for $\kappa_{n, \ell}^{2}$ even in the case when $n \approx 2^{\ell / 2}$. Remark that the mean number of visits $E N_{\lambda}$ can be $O(1 / n)$ !

The second case is the best possible: for every $\ell$ we have $\left\|f_{\ell}\right\|_{\infty}=1, f_{\ell}$ is canonical and $\|f\|_{D}^{2} v_{D}(F)=$ $\operatorname{Var}\left(Z_{\ell}\right)$.

b3) We consider now the general case in the main application:

Using the final assertions of Theorem 2, we see that we have asymptotically the same conclusion as in the case of b1: whenever $\ell$ is large, our $Z$, up to constants depending only on $\Phi$ and the law $p$, is the normalised $U$-statistic of order 2 corresponding to some canonical function the Sup norm of which is equivalent to 1.

Thus we get a large deviation inequality which cannot be obtained using the classical result for $2^{\ell / 2} \ll n \ll 2^{\ell}$.

\section{Proofs}

The proof is based on De la Pe $\tilde{n} a^{\prime} s$ inequalities [2]. As all bounds are continuous with respect to $\|f\|_{D}$, $v_{D}(F)$, it suffices to prove that, if $G$ is a collection such that $\Sigma_{i \in G} \operatorname{Sup}_{\lambda \in D} \operatorname{Sup}\left(f^{2}(x, y) I_{\lambda}(x) I_{\tau_{i}(\lambda)}(y)\right)=1$, then

$$
E\left(V_{n}^{2 k}\right) \leq C^{2 k} \times\left(\mathbb{E} \mathcal{N}^{2 k}\right)^{4} \times \operatorname{Max}\left(n^{2} v,\left(n^{2} v\right)^{k}\right)
$$

where, to simplify notations, we set

$$
V_{n}=\Sigma_{1 \leq i \neq j \leq n} \pi_{2} f\left(\xi_{i}, \xi_{j}\right) \text { and } v=v_{D}(F) .
$$

\section{1: Symmetrization}

Let $\varepsilon_{i}, \varepsilon_{i}^{\prime}, \mathcal{N}_{i}, \mathcal{N}_{i}^{\prime}, \xi_{i}, \eta_{i}$ be six independent n-samples: the common law of $\varepsilon$ 's is the law of the centered sign, the common law of the $\mathcal{N}$ 's is the normal $\mathcal{N}(0,1)$, the common law of $\xi$ 's and $\eta$ 's is the law $F$.

Using the first Theorem of De la Pe $\widetilde{n} a$, as $\pi_{2} f$ is canonical, we get:

For every $\Gamma$ even, increasing on $\mathbb{R}^{+}$and convex

$$
E \Gamma\left(V_{n}\right) \leq E \Gamma\left(4 \Sigma_{1 \leq i, j \leq n ; i \neq j} \pi_{2} f\left(\xi_{i}, \eta_{j}\right)\right) .
$$

Using the classical symmetrization inequalities (see [2] again), we have

$$
E \Gamma\left(V_{n}\right) \leq E \Gamma\left(16 \Sigma_{1 \leq i, j \leq n ; i \neq j} \varepsilon_{i} \varepsilon_{j}^{\prime} \pi_{2} f\left(\xi_{i}, \eta_{j}\right)\right) .
$$

As the $\varepsilon_{i}, \varepsilon_{i}^{\prime}$ can be viewed as conditional expectations of $\sqrt{\pi / 2} \mathcal{N}_{i}, \sqrt{\pi / 2} \mathcal{N}_{i}^{\prime}$, using convexity again we get

$$
E \Gamma\left(V_{n}\right) \leq E \Gamma\left(8 \pi \Sigma_{1 \leq i, j \leq n ; i \neq j} \mathcal{N}_{i} \mathcal{N}_{j}^{\prime} \pi_{2} f\left(\xi_{i}, \eta_{j}\right)\right) .
$$

We set now

$$
W_{n}=\Sigma_{1 \leq i, j \leq n ; i \neq j} \pi_{2} f\left(\xi_{i}, \eta_{j}\right)^{2} .
$$

In law, $\left.\left(\Sigma_{1 \leq i, j \leq n ; i \neq j} \mathcal{N}_{i} \mathcal{N}_{j}^{\prime} \pi_{2} f\left(\xi_{i}, \eta_{j}\right)\right)^{2}=\mathcal{N}^{2} \Sigma_{i}\left(\Sigma_{j} \pi_{2} f\left(\xi_{i}, \eta_{j}\right) \mathcal{N}_{j}\right)^{2}\right)$ or $\mathcal{N}^{2} \Sigma_{k} \lambda_{k} \mathcal{N}_{k}^{2}$, where $\Sigma_{k} \lambda_{k}=W_{n}$ with $\lambda_{k} \geq 0$, thus, by convexity:

Lemma 1. For every $k \in \mathbb{N}$, we have

$$
E V_{n}^{2 k} \leq(8 \pi)^{2 k}\left(E \mathcal{N}^{2 k}\right)^{2} E W_{n}^{k}
$$




\section{2: Bounds for functions}

$k$ is a natural integer. The current indexes $i, j$ of the sample belongs to $[1, n]$. The current $s$ belongs to $G$, other current indexes as $\lambda, \mu, .$. belong to $D$. We have $|f| \leq \sqrt{h}$ where

$$
h(x, y)=\Sigma_{\lambda, \mu} a_{\lambda, \mu} I_{\lambda}(x) I_{\mu}(y) .
$$

Thus $\left|\pi_{2} f(x, y)\right| \leq \iint(\sqrt{h}(x, y)+\sqrt{h}(x, t)+\sqrt{h}(z, y)+\sqrt{h}(z, t)) F(d z) F(d t)$ and finally

$$
\pi_{2} f(x, y)^{2} \leq 4 \int(h(x, y)+h(x, t)+h(z, y)+h(z, t)) F(d z) F(d t) .
$$

Thus by convexity

Lemma 2. For $h$ defined in (11) and natural integer $k$ we have

$$
E W_{n}^{k} \leq(16)^{k} E\left(\left(\Sigma_{1 \leq i \neq j \leq n} h\left(\xi_{i}, \eta_{j}\right)\right)^{k}\right) .
$$

\section{3: Bounds for moments}

We define the numbers of visits of $I_{\lambda}$ by each of the two samples as

$$
X_{\lambda}=\Sigma_{1 \leq i \leq n} I_{\lambda}\left(\xi_{i}\right) \text { and } Y_{\lambda}=\Sigma_{1 \leq i \leq n} I_{\lambda}\left(\eta_{i}\right) \cdot
$$

Let $\tau$ be the current permutation of $G$ and $\pi_{\tau}$ be $\operatorname{Sup}{ }_{\lambda} a_{\lambda, \tau(\lambda)}$. We have obviously

$$
\Sigma_{1 \leq i \neq j \leq n} h\left(\xi_{i}, \eta_{j}\right) \leq \Sigma_{\tau} \pi_{\tau}\left(\Sigma_{\lambda} X_{\lambda} Y_{\tau(\lambda)}\right) .
$$

As $\Sigma_{\tau} \pi_{\tau}=1$, by convexity again and (13) we obtain

$$
E W_{n}^{k} \leq(16)^{k} \operatorname{Sup}_{\tau} E\left(\left(\Sigma_{\lambda} X_{\lambda} Y_{\tau(\lambda)}\right)^{k}\right) .
$$

Appendix 1 contains the proof of the main technical result, namely:

Lemma 3. With previous notations, for every $\tau$, for every integer $k \geq 1$, we have

$$
E\left(\Sigma_{\lambda} X_{\lambda} Y_{\tau(\lambda)}\right)^{k} \leq 6^{k} \operatorname{Max}\left(n^{2} v,\left(n^{2} v\right)^{k}\right)\left(E \mathcal{N}^{2 k}\right)^{2} .
$$

Collecting the previous bounds, proof of Theorem 1 is achieved.

\section{4: Proof of Corollary 1}

A) We assume that $n^{2} v \geq 1$. Let $X$ be $U_{n}^{(2)}(f) / C\|f\|_{D} n \sqrt{v}$.

Appendix 2 contains the proof of the quite obvious

Lemma 4. If for every natural integer $k$ we have $\mathbb{E} X^{2 k} \leq\left(\mathbb{E} \mathcal{N}^{2 k}\right)^{4}$, then

$$
\mathbb{P}(|X| \geq x) \leq \exp (6-2 \sqrt{x}) .
$$

B) Now, if $n^{2} v \leq 1$, let $Y$ be $U_{n}^{(2)}(f) / C\|f\|_{D}$. For the same reason we have

$$
\mathbb{P}(|Y| \geq x) \leq \exp (6-2 \sqrt{x}) .
$$

This achieves the proof.

\section{5: Proof of Theorem 2}

Let $\Delta$ be some positive integer and $D$ be the partition $\left(I_{\lambda, D}=(\lambda / \Delta,(\lambda+1) / \Delta(\mid \lambda \in \mathbb{Z})\right.$. In what follows indexes $\lambda, u, v, s$ belong to $\mathbb{Z}$. 
Let $p_{\lambda, D}$ be $P\left(\xi \in I_{\lambda, D}\right)$ and $p_{D}$ be the density $p_{D}(x)=\Sigma \Delta p_{\lambda, D} I_{\lambda, D}$. Expectation with respect to $p_{D}$ is denoted $E_{D}$. We have

$$
\lim _{\Delta \rightarrow \infty}\left\|p_{D}-p\right\|_{2}=0, \Delta v_{D}=\left\|p_{D}\right\|_{2}^{2} \text { and thus } \lim _{\Delta \rightarrow \infty} \Delta v_{D}=\|p\|_{2}^{2}
$$

We set $\Phi(x)=\Sigma_{u \in \mathbb{Z}} \gamma_{u}(x-u) \mathbb{1}_{u \leq x<u+1}$, where the support of $\gamma_{u}$ is included in [0, $1\left[\right.$ (thus defining the $\gamma_{u}$ 's); we have $\left\|\gamma_{u}\right\|_{\infty} \leq \omega_{u}$. As $\|\Phi\|_{2}=1$, there exists some $u_{o}$ with $\left\|\gamma_{u_{o}}\right\|^{2}>0$.

We set $f_{D}(x, y)=\Sigma_{\lambda} \Phi(\Delta x+\lambda) \Phi(\Delta y+\lambda)$.

a) Bounds for $E f_{D}^{2}(\xi, \eta)$ and $\left\|f_{D}\right\|_{D}$ :

We begin by bounding from below the quantity $E f_{D}^{2}(\xi, \eta)$.

We have $E f_{D}^{2}(\xi, \eta)=\Sigma_{\lambda, \mu}(E \Phi(\Delta \xi+\lambda) \Phi(\Delta \eta+\mu))^{2} \geq \Sigma_{\lambda}\left(E \Phi^{2}(\Delta x+\lambda)\right)^{2}$, thus $E f_{D}^{2}(\xi, \eta) \geq \Sigma_{\lambda}\left(E \gamma_{u_{o}}^{2}(\Delta \xi+\right.$ $\left.\left.\lambda-u_{o}\right) I_{u_{o}-\lambda, D}\right)^{2}$. A classical computation gives $\left(E g I_{\mu, D}\right)^{2}-\left(E_{D} g I_{\mu, D}\right)^{2} \geq-2\left\|g I_{\mu, D}\right\|_{\infty}\left\|g I_{\mu, D}\right\|_{2} p_{\mu, D}\left(\int_{I_{\mu, D}}(p-\right.$ $\left.\left.p_{D}\right)^{2} d x\right)^{1 / 2}$ then $E f_{D}^{2}(\xi, \eta) \geq \Sigma_{\lambda}\left(E_{D} \gamma_{u_{o}}^{2}\left(\Delta \xi+\lambda-u_{o}\right) I_{u_{o}-\lambda, D}\right)^{2}-2 \omega_{u_{o}}\left(\left\|\gamma_{u_{o}}^{2}\right\|_{2} / \sqrt{\Delta}\right) \sqrt{v_{D}}\left\|p-p_{D}\right\|_{2}$. As $\left(E_{D} \gamma_{u_{o}}^{2}\right.$ $\left.\left(\Delta \xi+\lambda-u_{o}\right) I_{u_{o}-\lambda, D}\right)^{2}=p_{u_{o}-\lambda, D}^{2}\left\|\gamma_{u_{o}}\right\|_{2}^{4}$, we get

$$
\begin{gathered}
\Delta E f_{D}^{2}(\xi, \eta) \geq \Delta v_{D}\left\|\gamma_{u_{o}}\right\|_{2}^{4}-2 \omega_{u_{o}}^{3}\left\|p-p_{D}\right\|_{2} \sqrt{\Delta v_{D}} \text { and } \\
\lim \inf _{\Delta \rightarrow \infty} \Delta E f_{D}^{2}(\xi, \eta) \geq\left\|p_{2}\right\|^{2} a(\Phi):=\|p\|_{2}^{2}\left\|\gamma_{u_{o}}\right\|_{2}^{4}>0 .
\end{gathered}
$$

On the other hand, $\left|f_{D}(x, y)\right| \leq g(x, y):=\Sigma_{\lambda}|\Phi(\Delta x+\lambda) \Phi(\Delta y+\lambda)|$. Using $(\mathrm{H})$, we have $g(x, y) \leq \Sigma_{\lambda, u, v} \omega_{u+\lambda} \omega_{v+\lambda}$ $I_{u}(x) I_{v}(y)$.

Setting $\sqrt{a_{s}}=\Sigma_{u} \omega_{u} \omega_{u+s}, g(x, y) \leq \Sigma_{\lambda, s} \sqrt{a_{s}} I_{\lambda}(x) I_{\lambda+s}(y)$, then

$$
g^{2}(x, y) \leq \Sigma_{s} a_{s}\left(\Sigma_{\lambda} I_{\lambda}(x) I_{\lambda+s}(y)\right)
$$

But $\Sigma_{s} a_{s}=\Sigma_{u, v, s} \omega_{u} \omega_{v} \omega_{u+s} \omega_{v+s}=M(\Phi)^{2} \geq \Sigma_{u, v} \omega_{u} \omega_{v} \omega_{u} \omega_{v}=\left(\Sigma_{u} \omega_{u}^{2}\right)^{2} \geq 1$ because $\|\Phi\|_{2}=1$. Taking for $G$ the collection of $\lambda: \rightarrow \lambda+s$, we get

$$
\left\|f_{D}\right\|_{D}^{2} \leq M^{2}(\Phi) \text { and } M^{2}(\Phi) \geq 1
$$

We recall the obvious upper bound

$$
E f_{D}^{2} \leq v_{D} M^{2}(\Phi) \leq\|p\|_{2}^{2} M^{2}(\Phi) / \Delta
$$

b) Bounds for $\left\|f_{D}\right\|_{\infty}$ :

Obviously, $\left\|f_{D}\right\|_{\infty}$ does not depend on $D$, and is less than $M(\Phi)$ :

$$
\text { There exists some } b(\Phi) \text { with } 0<b(\Phi)=\left\|f_{D}\right\|_{\infty} \leq M(\Phi) \text {. }
$$

d) Bounds for $\left\|\pi_{2} f_{D}\right\|_{2}$ and $\left\|\pi_{2} f_{D}\right\|_{\infty}$ :

As for $\Delta$ large, $\operatorname{Sup}_{\lambda} p_{\lambda, D}=o(1 / \sqrt{\Delta})$ we have $|E \Phi(\Delta \eta+\lambda)|=o(1 / \sqrt{\Delta})$. Thus $\|E f(x, \eta)\|_{\infty}:=$ $\operatorname{Sup}_{x}|E f(x, \eta)| \rightarrow 0$. We have $\pi_{2} f_{D}(x, y)=f_{D}(x, y)-E f(x, \eta)-E f(\xi, y)+E f(\xi, \eta)$, and asymptotically we have

$$
\lim _{\Delta \rightarrow \infty}\left\|\pi_{2} f_{D}\right\|_{\infty}=b(\Phi) \text { (and obviously by (19) }\left\|\pi_{2} f_{D}\right\|_{\infty} \leq 4 M(\Phi) \text {. }
$$

We have $E\left(f_{D}\right)^{2} \geq \operatorname{Var}\left(\pi_{2} f_{D}\right) \geq \Sigma_{\lambda}\left(\operatorname{Var} \Phi^{2}(\Delta \xi+\lambda)\right)^{2}=\Sigma_{\lambda}\left(E\left(\Phi^{2}(\Delta \xi+\lambda)-E\left(\Phi(\Delta \xi+\lambda)^{2}\right)^{+}\right)^{2}\right.$. Using the fact that $\Delta E\left(\Phi(\Delta \xi+\lambda)^{2}\right.$ goes uniformly to 0 and (15), we get

$$
a(\Phi)\|p\|_{2}^{2} \leq \lim \inf _{\Delta \rightarrow \infty} \Delta \operatorname{Var}\left(\pi_{2} f_{D}\right)
$$


e) Proof of Theorem 2

$$
\Delta \operatorname{Var}\left(\pi_{2} f_{D}\right) \leq\|p\|_{2}^{2} M^{2}(\Phi)
$$

We set now $\Delta=2^{\ell}$. Using $n C / \sqrt{n /(n-1)} \leq 2 C, n^{2} v_{D} \geq 1,\left\|f_{D}\right\|_{D} \leq M(\Phi)$ and $\Delta v_{D} \leq\|p\|_{2}^{2}$, Corollary 1 gives the exponential upper bound.

\section{Appendix 1: Proof of Lemma 3}

In this appendix where partition $D$ is fixed, we use notation

$$
v_{D}=v, p_{\lambda}=P\left(\xi \in I_{\lambda}\right), \eta_{\lambda}=\mathbb{E}\left(X_{\lambda}\right)=n p_{\lambda} \text { thus } \Sigma \eta_{\lambda}^{2}=n^{2} v
$$

We recall that $\tau$ is some permutation of $D$, and that $\lambda$ is the current point of $D$.

We consider two laws on $\mathbb{N}^{D} \times \mathbb{N}^{D}$, the current point of which is $(\mathbf{X}, \mathbf{Y})$. In all cases, $\mathbf{X}$ and $\mathbf{Y}$ are independent with the same law.

In the first case, the law of $\mathbf{X}$ is $\mathcal{M}(n, \mathbf{p})(n \geq 2)$, the Multinomial where $\mathbf{p}=\left(p_{\lambda}\right)$, with associated expectation $E$.

In the second one, the $X_{\lambda}$ are independent, with Poisson law, and mean value $E_{\eta}(\mathbf{X})=\eta$, where $\eta=\left(\eta_{\lambda}\right)=$ $n \mathbf{p}$. The associated expectation is $\mathbb{E}_{\eta}$.

We consider the mapping $U$ from $\mathbb{N}^{D} \times \mathbb{N}^{D}$ to $\mathbb{R}$ defined by $U(\mathbf{X}, \mathbf{Y})=\Sigma_{\lambda} X_{\lambda} Y_{\sigma(\lambda)}$. We will first prove that for every positive integer $k E\left(U^{k}\right)$ is less than $E_{\eta}\left(U^{k}\right)$ and then furnish an upper bound for this moment.

\section{1: Reduction to the Poisson case}

In what follows, $E_{\mu}$ denotes the expectation associated to the Poisson law with parameter $\mu . X^{[k]}$ is the Polynomial $X(X-1) \cdots(X-k+1)$, for which $E_{\mu} X^{[k]}=\mu^{k}$.

Definitions: A mapping $\psi$ from $\mathbb{N}$ to $\mathbb{N}$ is strongly positive if

$$
\psi(X)=\Sigma_{k} a_{k} X^{[k]}, \text { with } a_{k} \geq 0 \text { for every } k .
$$

A mapping $\Psi$ from $\mathbb{N}^{D}$ to $\mathbb{N}$ is strongly positive if there exist some enumerable $I$, a family $\left(\psi_{\lambda, i} \mid \lambda ; i \in I\right)$, a family $\left(a_{i} \mid i \in I\right)$, where each $\psi_{\lambda, i}$ is strongly positive and each $a_{i}$ is positive, such that

$$
\Psi(\mathbf{X})=\Sigma_{i} a_{i} \Pi_{\lambda} \psi_{\lambda, i}\left(X_{\lambda}\right)
$$

Lemma 5. If $\Psi$ is strongly positive, then

$$
E(\Psi(\mathbf{X})) \leq E_{\eta}(\Psi(\mathbf{X}))
$$

For every $k, X^{k}$ and $\{X(X-1)\}^{k}$ are strongly positive. Moreover for $k>0$, we have

$$
E_{\mu}\{X(X-1)\}^{k} \leq \operatorname{Max}\left(\mu^{2 k}, \mu^{2}\right) \times\left(E \mathcal{N}^{2 k}\right)^{2} .
$$

Remark: The upper bound in (a2) is increasing of $k$ and $\mu(k \geq 1$ and $\mu>0)$.

Proof of (a1): By d2, it suffices to prove the formula when $\Psi(\mathbf{X})=\Pi X_{\lambda}^{\left[k_{\lambda}\right]}$. For such a $\Psi, E \Psi(\mathbf{X})=0$ if $\Sigma_{\lambda} k_{\lambda}>n$, and $\left(n ! /\left(n-\Sigma_{\lambda} k_{\lambda}\right) !\right) \Pi_{\lambda} p_{\lambda}^{k_{\lambda}}$ else, obviously less than $\Pi_{\lambda}\left(n p_{\lambda}\right)^{k_{\lambda}}=E_{\eta} \psi(\mathbf{X})$.

Proof of (a2): The fact that $X^{k}$ is strongly positive (in our sense) is well-known.

Let $T_{k}$ be $\{X(X-1)\}^{k} . T_{1}$ is $X^{[2]}$ and $E_{\mu} T_{1}=\mu^{2}$. Assume $k>1$; with $x=X-2, T_{k}=X^{[2]}\{(x+2)(x+$ $1)\}^{k-1}$. But $\{(x+2)(x+1)\}^{k-1}$ is polynomial with respect to $x$, with positive coefficients, thus strongly positive 
with respect to $x$ : $\{(x+2)(x+1)\}^{k-1}=\Sigma_{0 \leq j \leq 2 k-2} \gamma_{j, k} x^{[j]}$; finally $X^{[2]} x^{[j]}=X^{[j+2]}$ and $T^{k}$ is strongly positive. Moreover, $E_{\mu} T_{k}=E_{\mu} \Sigma_{0 \leq j \leq 2 k-2} \gamma_{j, k} X^{[2+j]}=\mu^{2} \Sigma_{0 \leq j \leq 2 k-2} \gamma_{j, k} E X^{[j]}=\mu^{2} E_{\mu}\{(X+2)(X+1)\}^{k-1}$.

Let $g_{k}$ be $g_{k}(X)=T_{k}(X) \mathbb{1}_{X>1} \cdot g_{k}$ is convex, and if $X$ is a Poisson r.v, almost surely $g_{k}(X)=T_{k}$. For $k=1$, $E_{\mu} g_{1}(X)=\mu^{2}$; let $k$ be $>1$. We have obtained $E_{\mu}\{X(X-1)\}^{k}=\mu^{2} E_{\mu} g_{k-1}(X+2)$. Let $Y$ be independent of $X$, Poisson with parameter 2 ; by Jensen, conditionally on $X=x, g_{k-1}(x+2) \leq E_{2} g_{k-1}(x+Y)$, thus, as the law of $X+Y$ is Poisson with parameter $2+\mu$, we get $E_{\mu}\{X(X-1)\}^{k} \leq \mu^{2} E_{\mu+2} g_{k-1}(X)$, thus, recursively

$$
\text { if } k>0 \text {, then } E_{\mu}\{X(X-1)\}^{k} \leq[\mu(\mu+2) \cdots(\mu+2 k-2)]^{2} .
$$

The product $\mu(\mu+2) \cdots(\mu+2 k-2)$ is bounded by $\operatorname{Max}\left(\mu^{k}, \mu\right) \times(1 \cdot 3 \cdot 5 \cdots(2 k-1))=\operatorname{Max}\left(\mu^{k}, \mu\right) \times E \mathcal{N}^{2 k}$ and the proof is achieved for a2.

Now we return to the proof. $U^{k}$ being a sum with positive coefficients of products of powers of the almost surely positive $X$ 's and the $Y$ 's is obviously strongly positive with respect to the $X$ 's and $Y$ 's; by independence and (a1), we obtain for every $k \geq 0$ :

$$
E U^{k} \leq E_{\eta} U^{k}
$$

\section{2: The Poisson case}

For every pair $x, y$ of natural integers, we have easily

$$
x y \leq x(x-1)+y(y-1)+\mathbb{1}_{x=1} \times \mathbb{1}_{y=1} .
$$

Let us define now

$$
\begin{aligned}
& Z=\Sigma_{\lambda} X_{\lambda}\left(X_{\lambda}-1\right), Z^{\prime}=\Sigma_{\lambda} Y_{\lambda}\left(Y_{\lambda}-1\right) \\
& T=\Sigma_{\lambda} \mathbb{1}_{X_{\lambda}=1} \times \mathbb{1}_{Y_{\tau(\lambda)}=1}
\end{aligned}
$$

Using the fact that $\tau$ is a permutation, by a4 we have $U \leq Z+Z^{\prime}+T$, then, as the laws of $Z$ and $Z^{\prime}$ are the same

$$
E_{\eta} U^{k} \leq 3^{k} \operatorname{Max}\left(E_{\eta} Z^{k}, E_{\eta} T^{k}\right)
$$

\section{3: Bound for the first term}

As $E_{\eta} Z=n^{2} v$, we assume that $k>1$. We set

$$
E(k, \mu)=\operatorname{Max}\left(\mu^{2 k}, \mu^{2}\right)\left(E \mathcal{N}^{2 k}\right)^{2} \text { if } k>0 \text { and } 1 \text { else }
$$

By a2, we have

$$
E_{\eta} Z^{k} \leq \Sigma_{k_{\lambda} \geq 0 ; \Sigma_{\lambda} k_{\lambda}=k}\left\{k ! / \Pi_{\lambda} k_{\lambda} !\right\} \Pi_{\lambda} E\left(k_{\lambda}, \eta_{\lambda}\right)
$$

First case: If for each $\lambda, \eta_{\lambda} \geq 1$, then, as $\Pi_{\lambda} E \mathcal{N}^{2 k_{\lambda}} \leq E \mathcal{N}^{2 \Sigma_{\lambda} k_{\lambda}}$, we have

$$
E_{\eta} Z^{k} \leq\left(E \mathcal{N}^{2 k}\right)^{2} \Sigma_{k_{\lambda} \geq 0 ; \Sigma_{\lambda} k_{\lambda}=k}\left\{k ! / \Pi_{\lambda} k_{\lambda} !\right\} \eta_{\lambda}^{2 k_{\lambda}}
$$

thus, in the first case, for every $k \geq 0$

$$
E_{\eta} Z^{k} \leq\left(n^{2} v\right)^{k}\left(E \mathcal{N}^{2 k}\right)^{2}
$$


Second case: For each $\lambda, \eta_{\lambda} \leq 1$ :

Let $A$ be a non-void subset of $\left[0, D\left[\cap \mathbb{Z}\right.\right.$, and $M(A, k)$ be the subset of $\mathbb{N}^{A}$ given by $\left(k_{i} \mid i \in A ; k_{i}>\right.$ 0 for each $\left.i \in A ; \Sigma_{i \in A} k_{i}=k\right)$. We set

$$
S(A, k)=\Sigma_{M(A, k)}\left\{k ! / \Pi_{i} k_{i} !\right\} \Pi_{i} E\left(k_{i}, \eta_{i}\right) .
$$

The general term of $S(A, k)$ is $\left\{k ! / \Pi_{i} k_{i} !\right\} \Pi_{i} \eta_{i}^{2} \Pi_{i} E\left(\mathcal{N}^{2 k_{i}}\right)^{2}$.

Let $\nu_{j}$ be $\left(E \mathcal{N}^{2 j}\right)^{2} / j !(j \in \mathbb{N})$. Elementary computation gives

$$
\text { If } 1 \leq j \leq k, \text { then } \nu_{j} \nu_{k} \leq \nu_{j-1} \nu_{k+1} .
$$

Thus the general term of $S(A, k)$ is bounded by $\Pi_{i} \eta_{i}^{2} E\left(\mathcal{N}^{2 k}\right)^{2}$ (obtained outside of $M(A, k)$, when all $k_{i}$ are 0 except one).

On the other hand, it is well-known that $|M(A, k)|=\left(\begin{array}{c}k-1 \\ a-1\end{array}\right)$, where $a=|A|$. Finally

$$
E_{\eta}\left(Z^{k}\right) \leq \Sigma_{\text {non void }} S(A, k) \leq\left(E\left(\mathcal{N}^{2 k}\right)^{2} \times\left\{\Sigma_{a>0}\left(\begin{array}{c}
k-1 \\
a-1
\end{array}\right)\left(\Sigma_{|A|=a} \Pi_{i \in A} \eta_{i}^{2}\right)\right\} .\right.
$$

As $\Sigma_{|A|=a} \Pi_{i \in A} \eta_{i}^{2} \leq\left(n^{2} v\right)^{a}$ and $\Sigma_{a>0}\left(\begin{array}{c}k-1 \\ a-1\end{array}\right)=2^{k-1}$, we get in the second case, for any $k \geq 1$

$$
E_{\eta} Z^{k} \leq 2^{k-1} \operatorname{Max}\left(n^{2} v,\left(n^{2} v\right)^{k}\right)\left(E \mathcal{N}^{2 k}\right)^{2} .
$$

General case: We divide $[0, D$ into two (non void) subsets:

$\Delta_{1}=\left(\lambda \mid \eta_{\lambda}<1\right)$ and $\Delta_{2}=\left(\lambda \mid \eta_{\lambda} \geq 1\right)$, and set $v_{i}=\Sigma_{\lambda \in \Delta_{i}} \eta_{\lambda}^{2}$. Using (A1, A2), we obtain

$$
E\left(Z^{k}\right) \leq\left(E \mathcal{N}^{2 k}\right)^{2}\left(n^{2} v_{2}\right)^{k}+\Sigma_{j>0}\left(\begin{array}{c}
k \\
j
\end{array}\right) 2^{j-1}\left(E \mathcal{N}^{2 j}\right)^{2}\left(E \mathcal{N}^{2 k-2 j}\right)^{2} \operatorname{Max}\left(n^{2} v_{1},\left(n^{2} v_{1}\right)^{j}\right)\left(n^{2} v_{2}\right)^{k-j} .
$$

The latter bound is increasing of $v_{i}$, each bounded by $v$. Thus finally, in any case, for $k \geq 1$

$$
E_{\eta} Z^{k} \leq 2^{k} \operatorname{Max}\left(n^{2} v,\left(n^{2} v\right)^{k}\right)\left(E \mathcal{N}^{2 k}\right)^{2} .
$$

\section{4: Bound for the second term}

We can bound $E_{\eta} \mathbb{1}_{X_{\lambda}=1} \times \mathbb{1}_{Y_{\tau(\lambda)}=1}$ by $b_{\lambda}^{2}:=\eta_{\lambda} \eta_{\tau(\lambda)}$. We take notations of Second case of previous paragraph. Setting now

$$
S^{\prime}(A, k)=\Sigma_{M(A, k)}\left\{k ! / \Pi_{i} k_{i} !\right\} \Pi_{i} b_{i}^{2} \text {, we have } E_{\eta} T^{k}=\Sigma_{A \text { non void }} S^{\prime}(A, k) .
$$

The current term of $S^{\prime}(A, k)$ is bounded by $k ! \Pi_{i} b_{i}^{2}$. Thus we obtain here

$$
E_{\eta} T^{k} \leq k ! 2^{k-1} \operatorname{Max}\left(w, w^{k}\right) \text { where } w=\Sigma_{\lambda} b_{\lambda}^{2} .
$$

As by Cauchy-Schwartz $w \leq n^{2} v$ and $k ! \leq\left(E \mathcal{N}^{2 k}\right)^{2}$, we get again for $k \geq 1$

$$
E_{\eta} T^{k} \leq 2^{k} \operatorname{Max}\left(n^{2} v,\left(n^{2} v\right)^{k}\right)\left(E \mathcal{N}^{2 k}\right)^{2} .
$$

Using (a3, a5, A3) and (A4), the proof is finished.

\section{Appendix2: Proof of Lemma 4}

For $k \in \mathbb{N}$, we set $u_{k}=e^{k} E \mathcal{N}^{2 k}(2 k+1)^{-k}, r_{k}=u_{k+1} / u_{k}=e\{(2 k+1) /(2 k+3)\}^{k+1}$ and finally $\varphi(x)$ $=1+(x+1) \log ((2 x+1) /(2 x+3))$ for $x \geq 0$, then $\varphi(k)=\log \left(r_{k}\right)$. We have

$$
\begin{aligned}
\varphi^{\prime}(x) & =\log ((2 x+1) /(2 x+3))+1 /(2 x+1)+1 /(2 x+3) . \\
\varphi^{\prime \prime}(x) / 2 & =1 /(2 x+1)-1 /(2 x+3)-1 /(2 x+1)^{2}-1 /(2 x+3)^{2} \leq 0 .
\end{aligned}
$$


As $\varphi^{\prime}$ goes to 0 when $x$ goes to $\infty$, we have $\varphi^{\prime} \geq 0$. As $\varphi$ goes to 0 when $x$ goes to $\infty, \varphi \leq 0$. Thus, for $k>0$, $u_{k+1} \leq u_{k} \leq u_{0}=1$ : we have proved that

$$
\text { for every } k \in \mathbb{N}, E \mathcal{N}^{2 k} \leq e^{-k}(2 k+1)^{k} \text {. }
$$

Let us assumme that for every $k \in \mathbb{N}, E X^{2 k} \leq\left(E \mathcal{N}^{2 k}\right)^{4}$. If $2 k+3 \geq \sqrt{x} \geq 2 k+1$, via Markov's inequality and assertion ${ }^{*}, P(|X| \geq x) \leq e^{-4 k} \leq e^{-2 \sqrt{x}+6}$. Then the result is proved for $x \geq 1$ and obvious for $0 \leq x \leq 1$.

\section{REFERENCES}

[1] M.A. Arcones and E. Giné, Limit Theorems for $U$-processes. Ann. Probab. 21 (1993) 1494-1542.

[2] V. De la Peña, Decoupling and Khintchine's inequalities for $U$-statistics. Ann. Probab. 20 (1992) 1887-1892.

[3] B. Laurent, Efficient estimation of integral functionals of a density. Ann. Statist. 24 (1996) 659-681.

[4] B. Laurent and P. Massart, Adaptative estimation of a quadratic functional by model selection (1998) preprint. 